\title{
Proximal Tibial Epiphyseal Fracture, Complete Separation: A Rare Occurrence
}

Arjun Markanday*, Gurumurthy B, Rupakumar CS, Adarsh T, Pramod BM and Harsimran Sidu

Department of Orthopaedics, JSS Hospital, Mysore, India

\begin{abstract}
Introduction: Dislocation of the knee joint and fractures about the proximal tibia are extremely rare occurrences in the paediatric age groups. Based on the Salter and Harris classification system, we present a case of Type 1 fracture of the proximal tibia with complete separation of the epiphysis. The case also highlights the presence of other concomitant injuries that accompany the trauma around the knee.

Case Report: An 8 year old male child was brought to the casualty of the hospital after sustaining injury to the knee while playing soccer at school. He was struck from front by another kid and immediately fell with extreme pain in the knee. He presented to us after a delay of 2 hours post injury. On arrival there was gross swelling of the knee joint and also the leg involving the foot. The distal pulsations were feeble but palpable. Toe movements were limited. Immediate radiological assessment revealed a fracture involving the proximal tibia. Patient was found to have signs of impending compartment syndrome. Emergency surgery was taken up in presence of a vascular surgeon, the fracture reduced, vascularity preserved and fasciotomy done. Child was discharged after a stay of 20 days with good range of movements at the knee joint.
\end{abstract}

Conclusion: The rarity of the proximal knee injuries, combined with neuro vascular damage render this area a perilous zone in paediatric orthopaedics. However prompt intervention leads to good functional recovery and early mobilization.

Keywords: Proximal tibial fractures; Salter and Harris; Closed reduction; Compartment syndrome

\section{Introduction}

Salter and Harris developed their classification of epiphyseal fractures in 1963 which was later expanded by Ogden et al. Normally in a Type 1 injury the fracture line extends through the physis however the complete epiphysis is rarely known to separate from the metaphysis.

The Salter and Harris classification is as follows (Figure 1).

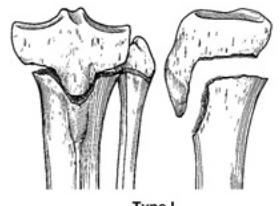

Type I

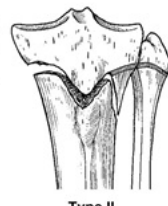

Type II

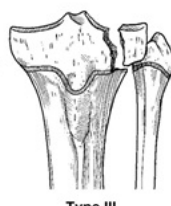

Type III

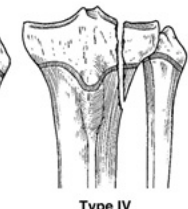

Type IV
Figure 1: The Salter and Harris classification.

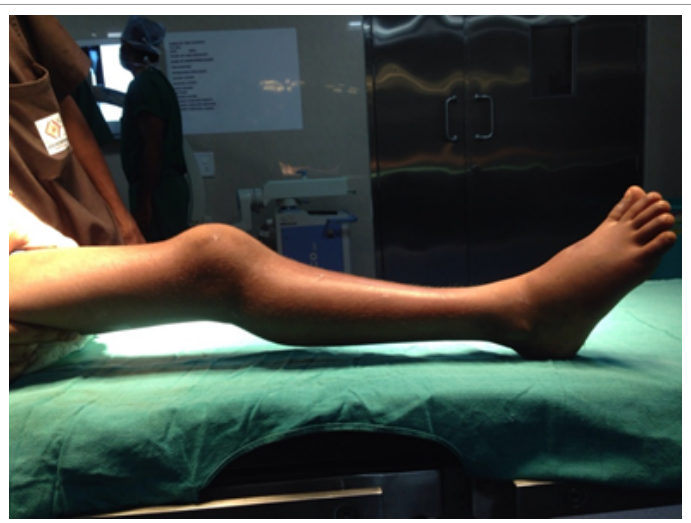

Figure 2: Pre Op appearance of the limb.
Prompt reduction usually leads to an uninhibited growth in the affected limb and rarely results in a limb length discrepancy. Fractures involving a complete separation of the epiphysis involve a considerable risk of preservation of the vascularity and the affect on limb length can only be affirmed after a long term follow up.

\section{Case Presentation}

An 8-year-old male child was brought to the casualty of the hospital after sustaining injury to the knee while playing soccer at school. $\mathrm{He}$ was struck from front by another kid and immediately fell with extreme pain in the knee. He presented to us after a delay of 2 hours post injury. On arrival there was gross swelling of the knee joint and also the leg involving the foot. The distal pulsations were feeble but palpable. Toe movements were limited. Immediate radiological assessment revealed a fracture involving the proximal tibia. Patient was found to have signs of impending compartment syndrome (pain to passive stretch, feeble dorsalis pedis pulsation, swelling of the entire leg). All the vital parameters were with in normal limits and he was fit to be taken up for surgery (Figures 2-4).

\section{Operative Procedure}

Under Spinal anesthesia an emergency Doppler scan of the popliteal region was done, this demonstrated a block in the popliteal artery. A

${ }^{*}$ Corresponding author: Arjun Markanday, Department of Orthopaedics, JSS Hospital, Mysore, India, Tel: 0821233 5555; E-mail: arjunmarkanday@yahoo.com

Received October 21, 2015; Accepted December 15, 2015; Published December 22, 2015

Citation: Markanday A, Gurumurthy B, Rupakumar CS, Adarsh T, Pramod BM, et al (2015) Proximal Tibial Epiphyseal Fracture, Complete Separation: A Rare Occurrence. J Trauma Treat 4: 277. doi:10.4172/2167-1222.1000277

Copyright: (c) 2015 Markanday A, et al. This is an open-access article distributed under the terms of the Creative Commons Attribution License, which permits unrestricted use, distribution, and reproduction in any medium, provided the original author and source are credited. 
vascular surgeon promptly removed the thrombus and reestablished the vascularity of the limb. We carried out a double incision fasciotomy as described by Mubarak and Hargens to relieve the pressure in the anterior and the posterior compartments. An anterolateral and a Posteromedial incision was used. Next the separated epiphysis was manually reduced under C-arm guidance. Once the correct positon of the epiphysis was ascertained $2 \mathrm{~K}$-wires were passed from lateral to medial making sure to avoid the common peroneal nerve and also ensuring that they after away from the articular surface of the knee so as to help in post-operative rehabilitation protocol. A long leg slab was applied to ensure immobilization after checking the correct position of the epiphysis under fluoroscopy.

The postoperative phase was uneventful and the signs of compartment syndrome resolved over a 48 -hour period. A second surgery for closing the fasciotomy incisions was taken up after 8

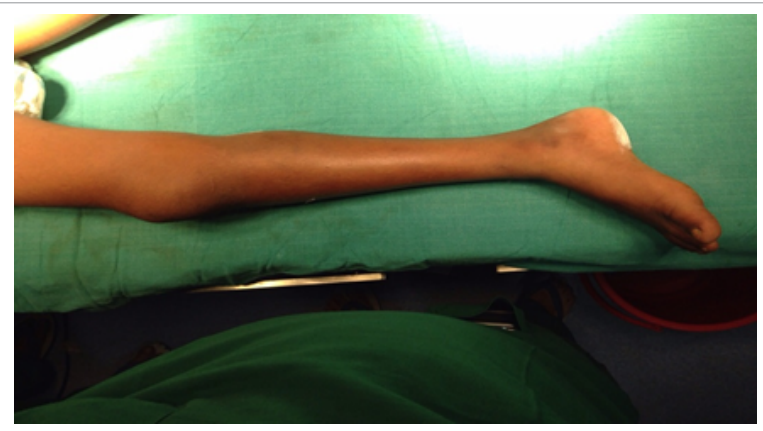

Figure 3: Pre Op Appearance of the limb.

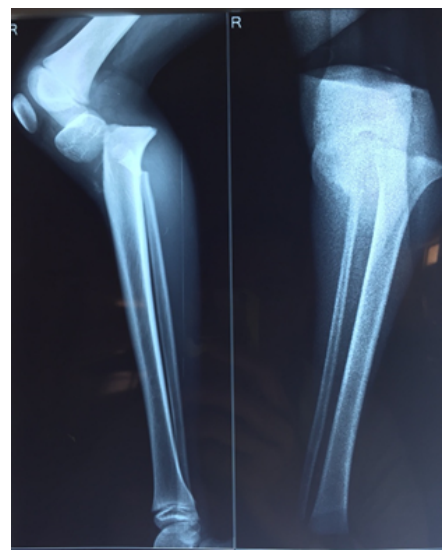

Figure 4: Pre Op X-Ray.

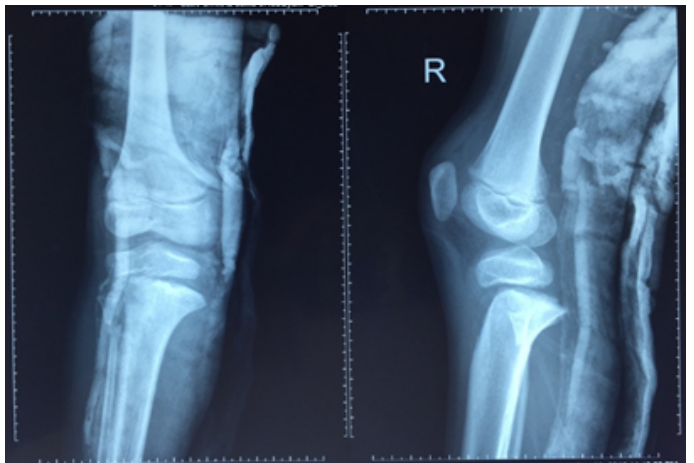

Figure 5: X-Ray after Slab Immobilization.

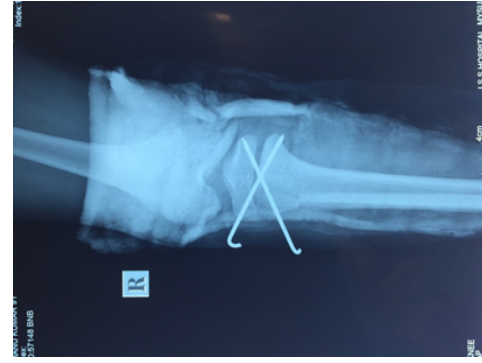

Figure 6: X-Ray after slab immobilization.

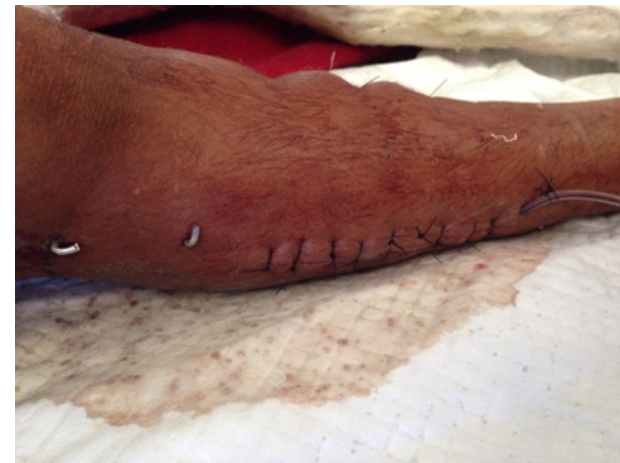

Figure 7: Post op day 2 appearance.

days of the first surgery using split skin grafts from the thigh. All the dressings were removed on the $14^{\text {th }}$ day after the initial surgery and a physiotherapy protocol involving active hip, knee and ankle range of movements were started. The child was discharged after 20 days without any complications (Figures 5-7).

\section{Discussion}

The myriad of problems that encompass the knee joint following trauma are well known to surgeons. Their prompt redressal is warranted in most cases. The threat of neuro vascular injury in the form of popliteal artery damage and peroneal nerve injury are imminent following this injury. Compartment syndrome has been widely discussed in many standard texts and journals however still it is the clinician who has to determine its presence in the absence of standard measuring instruments like the Whiteside's apparatus. Compartment syndrome diagnosis if delayed is known to lead to severe disability including the formation of contractures and ischemia. These finding often preclude the surgeons attention on the initial fracture. The complete separation of the epiphysis from the diaphysis also leads to a fear of the return of vascularity of the reduced fragment. This naturally leads to the constant threat of limb length discrepancy. Promptly reducing the displaced epiphysis has been known to ensure the survival of the epiphysis because the majority of the periosteal blood supply is still intact and which can ensure a proper growth provided the reduction is done in time. Also the work of the orthopaedician does not end after the surgery and one must ensure that a contentious physiotherapy program is carried out to ensure that no residual flexon deformity develops [1-6].

\section{Conclusion}

The fractures about the proximal tibia present with a lot of varied presentations, however the surgeon must ensure that equal attention is paid to the disabling conditions like compartment syndrome and vascularity as to the deformity. Timely reduction of the epiphysis has 
Citation: Markanday A, Gurumurthy B, Rupakumar CS, Adarsh T, Pramod BM, et al. (2015) Proximal Tibial Epiphyseal Fracture, Complete Separation: A Rare Occurrence. J Trauma Treat 4: 277. doi:10.4172/2167-1222.1000277

Page 3 of 3

been known to lead to a normal growth pattern. Also the need to work with a vascular surgeon is brought to the fore in cases that involve pressure symptoms due to the fracture fragments.

\section{References}

1. Lewis E Zionts (2002) Fractures around the knee in children. J Am Acad Orthop Surg 10: 345-355.

2. Mayer S, Albright JC, Stoneback JW (2015) Paediatric Knee Dislocations and Fractures about the knee. J Am Acad Orthop Surg 23: 571-580.
3. Watson-Jones R (1955) Fractures and joint injuries, Vol 2 (4thedn) Williams \& Wilkins, Baltimore, MD, USA.

4. Peterson HA (2007) Proximal tibia. In: Epiphyseal growth plate fractures. Springer, Berlin, 659 .

5. Zaricznyj B (1977) Avulsion fracture of the tibial eminence: treatment by open reduction and pinning. J Bone Joint Surg Am 59: 1111-1114.

6. Pape JM, Goulet JA, Hensinger RN (1993) Compartment syndrome complicating tibial tubercle avulsion. Clin Orthop Relat Res 295: 201-204. 